Sociologie et sociétés

\title{
Carnaval de Nice et carnavals indépendants
} Les mises en scène festives du spectacle de l'authentique

The Carnival of Nice and Independent Carnivals. Festive Settings for the Spectacle of the Authentic

\section{Christian Rinaudo}

Volume 37, numéro 1, printemps 2005

Le spectacle des villes

The Spectacle of Cities

URI : https://id.erudit.org/iderudit/012276ar

DOI : https://doi.org/10.7202/012276ar

Aller au sommaire du numéro

Éditeur(s)

Les Presses de l'Université de Montréal

ISSN

0038-030X (imprimé)

1492-1375 (numérique)

Découvrir la revue

Citer cet article

Rinaudo, C. (2005). Carnaval de Nice et carnavals indépendants : les mises en scène festives du spectacle de l'authentique. Sociologie et sociétés, 37(1), 55-68. https://doi.org/10.7202/012276ar

\section{Résumé de l'article}

En s'appuyant sur la notion d'imageidentifiante forgée par Marc Augé pour rendre compte des représentations que les collectivités locales donnent à voir de leur histoire, de leur patrimoine et de leur territoire à travers des discours sur « l'identité locale », cet article s'intéresse au Carnaval de Nice tel qu'il est impliqué depuis plus d'un siècle dans la construction touristique de l'image de la ville, à ses reformulations successives, à ses critiques ainsi qu'aux manifestations alternatives qui se sont développées ces dernières années sous la forme de " carnavals indépendants ». L'analyse souligne alors les tensions autour desquelles s'articulent les différents points de vue qui travaillent ces festivités comme "spectacle de l'authentique ». 


\section{Carnaval de Nice et carnavals indépendants.}

Les mises en scène festives du spectacle de l'authentique

\section{CHRISTIAN RINAUDO}

SOLIIS-URMIS (UMR 7032)

Université de Nice-Sophia Antipolis

24, avenue des Diables bleus

06357 Nice Cedex 4, France

Adresse électronique : rinaudo@unice.fr

D aradoxalement, à l'heure de la globalisation, le monde devient local(Bourdin, 2000), les signifiants identitaires localisés se multiplient alors même que les différentes régions du monde, et en particulier les grandes villes, ne cessent de développer leur degré d'interconnectivité (Hannertz, 1996). Cette dynamique identifiante des villes est d'autant plus affirmée qu'elle correspond à des logiques de développement et de transformation de l'industrie touristique. La relance des manifestations folkloriques et des fêtes populaires, l'élaboration de mythes locaux, la mise en cohérence d'un répertoire de symboles culturels à des fins touristiques sont des initiatives souvent citées en exemple du fait qu'elles conduisent à la mise en scène du patrimoine culturel ${ }^{1}$. Dans un autre registre, les festivals internationaux, biennales et autres événements culturels ou festifs sont encouragés par les collectivités en ce qu'ils contribuent à produire un ensemble d'images pour représenter une «identité locale». En France, de grandes manifestations culturelles telles que les Journées de Nantes, la Fiesta des Suds à Marseille, les Biennales de Lyon (d'art contemporain

1. Voir par exemple le travail réalisé par C. Davault (2000) sur les politiques de requalification symbolique des villes de Nantes et de Saint-Denis par les élites locales. Si l'histoire politique et du peuplement de ces deux villes est très différente, celles-ci ont cherché de la même façon à reconstruire leur image pour devenir des villes touristiques, culturelles, universitaires en inventant de nouvelles traditions locales d'accueil, d'hospitalité. 
et de danse), le Festival interceltique de Lorient, mais également certaines fêtes populaires comme les Férias de Nîmes et d'Arles, les fêtes de Bayonne (Garat, 1994) et les grands carnavals urbains comme ceux de Dunkerque et de Nice participent de cette dynamique.

Le Carnaval de Nice représente de ce point de vue un objet d'analyse particulièrement intéressant. Impliqué depuis plus d'un siècle dans la construction touristique de l'image de la ville, il est aujourd'hui au centre de diverses mises en cause qui sont révélatrices des tensions autour desquelles s'élaborent les discours sur l'identité locale. Pris en charge en 1873 par le Comité des fêtes dans le but de développer le tourisme d'hiver déjà bien implanté dans la région, il fait depuis quelques années l'objet de diverses contestations publiques de la part d'associations traditionalistes ou de collectifs d'artistes qui proposent des visions alternatives de la mise en scène festive de la ville.

Afin de décrire les modalités de mise en spectacle de Nice à travers son carnaval, nous utiliserons, à la suite de Marc Augé, la notion d'image identifiante pour désigner les images produites pour représenter une «identité locale», un patrimoine, une histoire, un territoire, de l'«authenticité», un dynamisme culturel:

Les images «identifiantes» sont aujourd'hui l'équivalent des images «édifiantes» d'hier. Il ne s'agit plus «d'édifier» des individus, de les instruire, de les construire, pour les identifier progressivement à l'idéal chrétien et moral partagé, mais d'identifier des collectivités, de les enraciner dans l'histoire, de conforter et d'asseoir leur image, de les mythifier pour que les individus à leur tour puissent s'y identifier. (Augé, 1994, p. 107)

Ainsi conceptualisée, la notion d'image identifiante nous servira ici à désigner l'ensemble des images et des discours qui organisent la mise en scène festive de l'identité à travers les brochures touristiques, les articles de la presse locale et nationale, l'organisation et le déroulement concret des manifestations et des contre-manifestations, les symboles qu'elles véhiculent, etc. Bref, tout ce qui, selon la formule de S. Cousin (2003), «tend à produire une image normée à laquelle chacun est prié de s’identifier».

\section{RELANCE DES TRADITIONS CARNAVALESQUES: LE LOCAL INSTRUMENTALISÉ}

Historiens et folkloristes locaux renvoient généralement les origines du Carnaval de Nice à un passé immémorial et font remonter ses traces les plus anciennes à la fin du XIII siècle². Après de multiples évolutions, c'est le Comité des fêtes de Nice, créé pour l'occasion en 1873, qui est chargé d'organiser les festivités sous le patronage de la municipalité. On est alors à l'époque de la construction de la "Côte d'Azur», lieu de villégiature d'hiver pour les monarchies et l'aristocratie européennes depuis l'extension jusqu'à Nice de la ligne de chemin de fer, achevée en 1864. Le terme même de "Côte d'Azur ", que les Américains traduiront plus tard par l'expression «French Riviera », est le produit d'une création externe, du regard touristique qui, depuis lors, n'a cessé de jouer un rôle important dans la construction identitaire de la ville de Nice.

2. Voir par exemple C.-A. Figuiera (1985). 
Dans ce contexte, le carnaval constituait le moment culminant de la saison d'hiver pour ces hivernants à la recherche de soleil et de divertissements. Sa Majesté Carnaval était alors montée sur des chars de plus en plus imposants pour devenir un personnage géant, à l'image d'une ville en plein essor. Sa propre représentation a évolué du personnage gargantuesque malicieux et fêtard, habillé en tenue de paysan ou de pêcheur, vers celle d'un Roi-Soleil majestueux donnant une image "d'éclat et de lumière » plus flatteuse pour la ville et pour ses qualités touristiques. Les pressions exercées par le Comité des fêtes pour éliminer toute allusion localiste à la culture populaire niçoise pouvant choquer ou «ennuyer» le public étranger sont alors très significatives de cette volonté de contrôler l'image d'un personnage carnavalesque présenté comme un conquérant du soleil et de la féerie. En 1939 par exemple, le secrétaire général du Comité des fêtes adressait un courrier au maquettiste G.-A. Mossa, chargé de la conception des chars, pour lui demander de remplacer certains attributs de la «culture niçoise» qui entouraient le monarque et qui risquaient d'apparaître comme « un peu trop local pour les étrangers ${ }^{3} »$.

Ainsi, la dimension locale du carnaval devait se retirer progressivement des cortèges qui, par les thématiques abordées et par les mises en scène de plus en plus cosmopolites, prenaient la forme d'un grand spectacle destiné à divertir le public mondain d'une Côte d'Azur érigée en capitale d'un tourisme de prestige. Cette orientation a été renforcée par l'installation de barricades tout le long du parcours et de tribunes composées de places payantes pour les spectateurs les plus aisés. Elle fut également confortée par l'instauration des batailles de fleurs se voulant plus raffinées et plus poétiques que les corsos carnavalesques, mais également plus en phase avec l'image féerique produite par les guides touristiques de l'époque ${ }^{4}$.

Si cette logique de modernisation du carnaval n'a cessé de se poursuivre depuis lors, elle a dû également s'adapter au fil des décennies à la démocratisation du tourisme et aux nouvelles exigences d'un public toujours plus nombreux attiré sur les rivages de la Côte d'Azur. Dans les années 1950 et 1960, Nice accueillait plus d'un demi-million de touristes chaque année, mais sa fonction de ville de repos et de retraite commençait également à s'affirmer. La saison hivernale, amputée de ce qui constituait jusque dans les années 1930 la société cosmopolite des hivernants, ne devait plus guère survivre que grâce au séjour climatique des personnes âgées, aux batailles de fleurs et à un carnaval qui, dans ce contexte, prenait l'allure d'un spectacle pour retraités où le public restait figé dans les tribunes et où les mannequins carnavalesques essayaient de recréer des mouvements artificiels grâce à l'élaboration de tout un système de mécanisation.

Plus récemment encore, l'organisation des festivités a été confiée à la direction générale de l'Office du tourisme et des congrès de Nice (отСN), ce qui devait marquer symboliquement l'aboutissement d'un processus visant à faire de cet événement un des moteurs

3. Cité dans A. Sidro (1979a).

4. Voir S. Liégeard (1988). Conçu comme un récit de voyage, cet ouvrage, dont la première édition date de 1887, a largement contribué à diffuser l'imagerie et le terme même de Côte d'Azur dans la société mondaine de l'époque. 
du développement économique de la ville centré sur l'industrie des loisirs et du tourisme 5 . Mais dans cette optique, la municipalité a également cherché à rajeunir l'image du carnaval de manière à attirer dans la ville un public plus dynamique, plus enclin à faire la fête et à investir les nouveaux espaces commerciaux du Vieux-Nice (petites boutiques de mode, restaurants, bars...) qui représentent, à côté des magasins de souvenirs fréquentés par la clientèle voyagistes, une source importante de retombées économiques pour la ville ${ }^{6}$. Pour le directeur de l'oтсN, le Carnaval de Nice est d'abord une affaire de marketing qui consiste à faire venir toujours plus de monde sur la Côte d'Azur et à diversifier la clientèle: «Il n'y a pas de raison que l'événement soit réservé à une clientèle monoculturelle à basse contribution comme c'est encore le cas. Nous ciblons à présent une clientèle diversifiée: jeunes, troisième âge, hommes d'affaires, etc. » Cela impliquait notamment, comme il le précise, l'introduction de commanditaires privés pour organiser des concerts et des techno parades, l'installation de «villages viP» pour ceux qui «dépensent » et qui sont prêts à investir pour bénéficier de services et d'espaces particuliers, mais également de s'entourer de personnalités du monde du spectacle chargées de trouver de nouvelles formes d'expression de la fête. Ainsi, lors de l'édition 2000, le recrutement du dessinateur Serguei ${ }^{7}$ comme « $y$-magier officiel» - le terme est en soit plus «branché » que celui de maquettiste - participait de cette volonté, selon la rhétorique de l'отсN, de « relooker» le carnaval. "Les grosses têtes d'antan sont l'expression d'une "manifestation terroir" qui n'attire personne et qui ne fait pas vendre le carnaval hors de Nice», expliquait le directeur de l'отсN. «Sergueï a fait partie de notre démarche électrochoc que nous voulons et devons prolonger afin d'obtenir la médiatisation record de cette année ${ }^{8}$. »

Dans son Carnaval 2000, Roi des Odyssées.com, Sergueï développa une symbolique se voulant plus universelle que celle des carnavaliers niçois qui s'inspiraient encore des dessins d'Alexis Mossa (1844-1926) et de son fils Gustav-Adolf Mossa (1883-1971), maquettistes

5. Aujourd'hui, la part des emplois afférente au tourisme (plus de un sur trois) témoigne du rôle prédominant de cette fonction dans l'économie de la région. Selon le Riviera Tourism Board, la Côte d'Azur accueille chaque année 9,8 millions de touristes dont $58 \%$ viennent de l'étranger, ce qui représente un apport financier global de 5,6 billions d'euros et qui permet d'assurer la viabilité de quelque 26 ooo emplois dans les secteurs des services et du commerce.

6. Situé à deux pas de la mer, le Vieux-Nice fait l'objet d'une attention particulière dans tous les guides touristiques. On y célèbre son cachet, ses façades en trompe-l'œil, son « authenticité». Depuis sa réhabilitation sociale, cet ancien quartier populaire ne cesse de s'imposer localement comme le lieu d'activités festives et culturelles de la ville. Galeries d'art, restaurants « typiques» ou « exotiques», terrasses de café, boutiques de mode, magasins de souvenirs ont fleuri dans les ruelles étroites, autour des petites places ensoleillées et le long du cours piétonnier qui le traverse. Tout cela a contribué à faire du Vieux-Nice le lieu de balade privilégié des touristes et à séduire une population de plus en plus composée d'étudiants et de jeunes artistes qui en ont fait, comme on dit, un quartier très «branché».

7. Serguei est notamment connu pour ses dessins humoristiques publiés dans Le Monde, L'Express ou le New York Times.

8. Cité dans Nice-Matin du 20 mars 2000, «Maintenir "l'effet Serguei" ». 
officiels du Carnaval de Nice pendant près d'un siècle 9 . En abordant des thèmes comme les droits de l'homme, la connaissance, la communication, la consommation, les transports, les nouvelles technologies, il permettait à un public plus large de comprendre les messages et participait du processus de délocalisation du carnaval entamé lors de sa prise en charge municipale, en rompant avec le jeu sur les signifiants niçois qu'avaient maintenus subrepticement le père et le fils Mossa malgré les injonctions du Comité des fêtes comme l'ont bien analysé les historiens locaux ${ }^{10}$.

Or, cette délocalisation des symboles du Carnaval de Nice devait s'accompagner d'un processus corollaire de relocalisation dont le but était de répondre à la quête d'authenticité des touristes qui évoluent dans un univers de plus en plus uniformisé et qui attendent d'une manifestation locale qu'elle exhibe les signes de son particularisme de manière à proposer au monde un certain exotisme, une «couleur locale».

Il s'agissait alors d'introduire dans une manifestation globale des éléments conventionnels et compréhensibles par tous du particularisme local et non plus de glisser de façon insidieuse des signifiants ésotériques de l'identité. C'est dans cette optique qu'une autre initiative de la municipalité a été de constituer une équipe "d'agitateurs professionnels »-également appelés « carnavaleurs» - et dont la mission était de susciter l'intérêt d'une clientèle en quête d'expériences festives et artistiques plus «authentiques ». Composée de jongleurs, d'acrobates, de danseurs et de comédiens placés sous la direction d'un " concepteur d'événements ${ }^{11}$ ", cette équipe a été chargée d'organiser des spectacles de rue qui devaient renouer les liens avec le passé et avec la tradition carnavalesque de Nice. Le «Grand Charivari », organisé lors de l'édition 2000, était au centre de cette nouvelle dynamique. Il fut présenté dans les brochures de l'oтсN comme « un air de folie issu des coutumes festives d'antan ». On pouvait y apprendre qu'il existait dans un passé lointain lorsque les jeunes munis de divers ustensiles et d'instruments à percussion allaient faire du vacarme devant la maison des veufs ou des veuves qui se remariaient sans s'être acquittés de la taxe du «Charavilh » à «l'abbé des fous ». On y découvrait également, grâce aux explications d'une ethnologue et historienne du Carnaval de Nice sollicitée pour

9. Tous deux furent peintres, aquarellistes, conservateurs du Musée des beaux-arts de Nice et, à tour de rôle, «maquettistes du Roy». «Alexis Mossa, écrit une historienne du Carnaval de Nice, est le premier père spirituel de Sa Majesté Carnaval. Il organisa les cortèges d'arrivée, construisit les chars dans les chantiers de la rue Miron, créa et publia l'album de Carnaval et donna naissance à Madame Carnaval en 1893. Aquarelliste de talent, il a ouvert tout naturellement la voie à son fils Gustave-Adolf, qui insuffla une vie riche et variée, non seulement à Carnaval, mais à tout le cortège.» (Sidro, 1979b)

10. A. Sidro (1979a) par exemple: «G.-A. Mossa, en grand connaisseur du folklore et du pays niçois, ne manquait jamais de faire paraître un ravioli ou un cougourdon ou le bouffon "Chicastrassa" aux côtés de son fils spirituel, même s’il devait parfois subir les reproches épistolaires de représentants du Comité des Fêtes plus soucieux de la compréhension d'un public “étranger” à la culture locale que de l'expression d'un art populaire, compris des seuls initiés, avec parfois le malin plaisir que l'on peut imaginer.» C.-A. Fighiera (1973) également: «Ce qui plaît aux Niçois, c'est que le carnaval garde un caractère local. C’est pour eux une joie de déchiffrer dans le défilé une inscription en dialecte, ou de découvrir dans la décoration d'un char un détail de mise en scène où leur perspicacité peut retrouver la petite patrie. »

11. Il s'agit de Gad Weil, à qui l'on doit notamment la transformation des Champs-Élysées en champ de blé ou l'organisation de la Techno Parade de Paris. 
collaborer à l'organisation de la relance des charivaris, que ceux-ci avaient au Xvi ${ }^{e}$ siècle une fonction sociale de lutte contre la corruption lorsque, par exemple, on allait faire un charivari devant la maison d'un notable accusé de détournement de fonds.

En ce sens, si le Grand Charivari pouvait apparaître, dans la logique de l'oтcN, comme un outil promotionnel en phase avec les exigences du tourisme moderne, il était aussi une manière d'anticiper la critique de ceux qui n'allaient pas manquer de s'indigner de la transformation du Carnaval de Nice en produit de consommation culturelle coupé de la tradition et de son ancrage local.

\section{SURVIVANCE DES TRADITIONS ANCESTRALES : LE LOCAL ESSENTIALISÉ}

Depuis longtemps, historiens, folkloristes et journalistes constatent, dénoncent ou se plaignent de la perte de signification du Carnaval de Nice qui, comme on peut souvent le lire, se serait coupé de «ses anciennes racines populaires ${ }^{12}$ ", perdant du même coup de son «authenticité». De ce point de vue, la dynamique de modernisation impulsée lors de l'édition 2000 avec le recrutement de Sergueï a eu pour effet de susciter de vives réactions de la part d'une critique à visée "traditionaliste» qui émanait des associations folkloriques et des sociétés de carnavaliers niçois qui se chargent de la construction des chars.

Dans le contexte du Carnaval de Nice, la «tradition locale» de laquelle se réclament les auteurs de la critique « traditionaliste » du carnaval est celle qui renvoie à la fabrication des chars par des sociétés d'artisans qui, depuis 1923, travaillent pour le compte de la municipalité $^{13}$. Il est souvent signalé à leur sujet, comme ici dans le supplément d'un journal local consacré au carnaval, que ces artisans sont « tous Niçois, tous carnavaliers de père en fils» et qu'ils se «transmettent le savoir-faire au fil des générations ». C'est donc bien au nom de la conservation d'une «tradition» supposée réelle et dans le but de se conformer à des normes immémoriales - celles qui justifient, précisément au nom de la tradition, l'exclusion de personnes étrangères au cercle fermé des carnavaliers niçois - que se compose cette posture traditionaliste. Elle se réclame en particulier de l'héritage des Mossa, maquettistes de père en fils jusqu'en 1971, qui ont puisé dans le passé pour forger un style que les associations traditionalistes revendiquent aujourd'hui comme étant l'image de marque du Carnaval de Nice. Il s'agit d'une forme d'expression populaire qui, comme l'a bien montré Bakhtine (1970) dans ses travaux sur Rabelais, s'appuie sur le grotesque et sur la bouffonnerie des personnages plutôt que sur un esthétisme classique, et qui a contribué ici à créer un monde de géants hilares, de grosses têtes aux couleurs criardes, de guirlandes multicolores dessinant sur les façades monumentales de la place principale de la ville toutes sortes de personnages obèses, vaudevillesques, à la limite de l'obscénité.

Dans ces conditions, la tentative de Sergueï de revenir à une forme d'expression plus allégorique telle qu'elle existait également dans le passé introduisait une rupture du point de vue des gardiens de cette «tradition». Les chars du Carnaval 2000 ont été critiqués

12. Nice-Matin du 23 février 2000.

13. Jusqu'en 1997, il y avait quatre associations dont chacune avait la charge d'une quantité de chars et de grosses têtes et recevait une subvention de la municipalité. Aujourd'hui, ce sont six entreprises familiales qui répondent chaque année à l'appel d'offre lancé par l'oTCN et qui se répartissent l'ensemble des réalisations. 
comme étant « riquiqui, chétifs, nés de l'intellectualisme torturé et parisianniste d'esprits contemporains soi-disant décorateurs ${ }^{14} »$. «Tout est uniforme», expliquait le représentant d'une association de défense des traditions, «tout est tellement bleu que c'en devient gris. Où sont les grosses têtes hilares, les chars de lumière, les grimaces de carton-pâte? Où est notre tradition ${ }^{15}$ ? " Le quotidien Nice-Matin parlait alors de la «Fronde "nissarde" contre le carnaval Sergueï ${ }^{16}$ » et ouvrait une tribune à ces "défenseurs de l'identité culturelle niçoise » qui «crient tous au crime de lèse-tradition ${ }^{17}$ ».

Ainsi, le « relookage » du Carnaval de Nice s'est transformé sous l'impulsion de cette critique traditionaliste aux accents conservateurs, anti-centralistes et anti-intellectualistes en une "affaire Sergueï», en un événement public centré sur la mise en question de l'identité culturelle niçoise. Ses auteurs reprochaient aux organisateurs et, en tout premier lieu, au maire de Nice d'être allé chercher « un Parisien, à savoir Sergueï, qui, par essence, ne connaît rien à nos traditions festives, pour concevoir notre carnaval ${ }^{18}{ }^{\prime}$. L'affaire a pris une telle ampleur médiatique que le maire s'est vu dans l'obligation de défendre son choix initial tout en revenant sur une position susceptible de satisfaire les associations traditionalistes sur lesquelles il s'est largement appuyé par ailleurs pour asseoir sa légitimité électorale, n'étant pas lui-même d'origine niçoise: «Il fallait secouer les puces de ce carnaval qui ressemblait de plus en plus à un spectacle de télévision. Il fallait frapper un grand coup. Et c'est fait. (...) Mais on verra alors s'il ne faut pas revenir en 2001 à un concept moins innovant et choquant ${ }^{19}$.» C'est précisément ce qu'il fit en annonçant quelques mois plus tard que les carnavaliers allaient retrouver leurs rôles habituels lors de l'édition 2001.

Le Grand Charivari introduit lors de l'édition 2000 en même temps que la modernisation du carnaval que devait incarner Sergueï n'était pas de nature à apaiser la critique. Pour les gardiens de la tradition carnavalesque, cette initiative de la municipalité et de l'отсN n'œuvrait pas à un retour à l'authenticité perdue, elle visait à répondre à la quête d'authenticité des touristes par une mise en scène de celle-ci. Elle ne représentait donc, de leur point de vue, qu'un outil de la promotion du carnaval et de la ville de Nice sur la scène mondiale, une tradition revisitée - et, de leur point de vue, «dénaturée »-permettant de répondre à la quête d'authenticité des touristes sans pour autant répondre aux exigences d'authenticité des « Niçois».

D'une certaine manière, cette façon de défendre une identité «pour soi» - née d'une socialisation commune et des expériences collectives soutenues par une langue, des traditions et un folklore transmis de génération en génération -, distincte de l'identité «pour les touristes » considérée comme factice, inauthentique, inessentielle, est une manière de perpétuer la tension entre la société locale des « vrais Niçois» et la société cosmopolite des riches « hivernants » autour de laquelle devait se construire l'identité de la ville à la fin du $\mathrm{XIX}^{\mathrm{e}}$ siècle du fait de la notoriété mondiale et de la contestation locale de la notion de Côte

14. Supplément «Spécial Carnaval», Nice-Matin, février-mars 2000.

15. Cité dans Nice-Matin du 16 février 2000, "Carnaval: polémique identitaire ».

16. Nice-Matin du 16 février 2000 (première page).

17. Nice-Matin du 16 février 2000, «Nice, le carnaval dans la tourmente identitaire?»

18. Ibid.

19. Cité dans Nice-Matin du 18 février 2000, «Jacques Peyrat: “J'ai trouvé plus conservateur que moi”.» 
d'Azur. C'est en effet à cette époque, peu de temps après le rattachement du comté de Nice à la France, survenu en 1860 par voie de référendum, que s'est développé ce point de vue localiste et traditionaliste qui contrastait avec l'indifférence affichée de la société mondaine et cosmopolite à l'égard de la population autochtone et s'attachait à défendre une identité «nissarde» menacée. La position essentialiste défendue reposait sur une opposition entre les «Niçois de souche» alors qualifiés de «Nissarts» et les autres, «hivernants», simples touristes de passage ou nouveaux habitants venus s'installer définitivement dans la ville mais ne pouvant être d'une origine locale. Ainsi, la résurgence de la «fronde nissarde » comme elle fut qualifiée lors de l'édition 2000 du Carnaval de Nice à propos de «l'affaire Sergueï» est symptomatique de cette tension toujours persistante autour de l'identité de la ville, des conditions et des enjeux de sa production sociale.

\section{L'INVENTION DES CARNAVALS «INDÉPENDANTS 》: LE LOCAL IMAGINÉ}

Depuis quelques années, une autre forme de critique du Carnaval de Nice commence à se faire entendre, suscitant de plus en plus d'intérêt dans les milieux alternatifs de la région. Elle émane d'acteurs résolus à s'inventer une place dans leur ville, des jeunes issus de milieux sociaux plutôt aisés pour la plupart d'entre eux, mais refusant de rentrer dans une logique de reproduction d'une classe moyenne ascendante. Confrontés au déficit de lieux de création, d'expression et de rencontre, ceux-ci militent activement pour une reconquête de la ville qui est passée par l'occupation, d'abord illégale puis régularisée grâce au soutien de l'État, de friches militaires situées dans un quartier populaire de Nice et par l'implantation sur ce site de collectifs au fonctionnement libertaire et dont les projets d'ordre public associaient des dynamiques artistiques, culturelles et sociales. Comme le souligne un récent rapport du ministère de la Culture qui s'est intéressé à leurs actions, « cette initiative s'inscrit dans un contexte niçois particulièrement difficile où le travail mené par le secteur culturel et social en matière associative est peu ou pas reconnu par les institutions » (Lextrait, 2001). Considérant leur démarche comme légitime, les membres des collectifs, rassemblés dans ce lieu de friche et militant contre sa démolition programmée ou pour qu'un autre espace plus pérenne leur soit confié, sont entrés dans un rapport de force avec le principal interlocuteur local, la municipalité, qui refuse de les reconnaître publiquement tout en étant contraint de devoir composer avec leurs revendications de démocratie locale. Ils s'en prennent tout particulièrement à la politique culturelle de la ville consacrée au prestige et privilégiant une démarche muséographique de l'art conforme à la demande du tourisme culturel mais peu enclin à cultiver d'autres modes de rapport au monde que celui de sa mise en spectacle instrumentalisée.

Ces initiatives locales participent d'un mouvement plus général bien analysé par Boltanski et Chiapello dans Le nouvel esprit du capitalisme (1999), de résurgence d'une critique «artiste» centrée sur la dénonciation de la mise en scène de la différence à des fins marchandes:

La marchandisation de tout, comme appropriation capitaliste de la différence pour en tirer un profit, peut-elle, aussi bien être dénoncée [...] comme mise en spectacle de tout, comme anéantissement de tout élan vital authentique qui, à peine esquissé, est immédiatement encodé afin de prendre place dans la circulation marchande des signes qui se substituent alors 
à l'expérience de la «vie» véritable au contact de monde. [...] L'autocritique capitaliste de la société de masse et la marchandisation de la différence ont ouvert la voie à une dénonciation de la réalité tout entière comme illusion et comme mise en scène: comme spectacle en tant que forme ultime de marchandise. (p. 445-446)

Or, comme le soulignent ces auteurs, l'aporie sur laquelle butent les entreprises de déconstruction de la notion d'authenticité et sa critique corollaire du monde comme spectacle est qu'elle sape toute position normative et cognitive à partir de laquelle une telle dénonciation peut être posée:

La visée radicale de la nouvelle critique menace sans arrêt sa propre position d'énonciation car on ne peut pas ne pas se demander d'où l'optique critique peut être adoptée si tout n'est que simulacre et que spectacle. (p. 552)

C'est alors, comme le soulignent Boltanski et Chiapello, en limitant l'extension de la sphère marchande par la création d'espaces, d'activités et d'artefacts qui tentent de s'en extraire que se régénère aujourd'hui la nouvelle critique artiste. Cela concerne non seulement les productions artistiques mais, plus globalement, toute forme de biens que l'on considère comme dégradés par leur transformation en produits proposés sur un marché concurrentiel et parmi lesquels les festivals d'été, les carnavals, les produits du terroir ainsi que certains lieux de la ville comme les centres historiques se trouvent particulièrement exposés du fait de leur mise en valeur dans le cadre des politiques culturelles et touristiques des villes.

Lors de l'édition 2000 du Carnaval de Nice, les auteurs de cette critique, membres des collectifs artistiques et culturels établis dans les lieux de friche ne visaient pas, à l'instar de la posture traditionaliste, à œuvrer au rétablissement d'une quelconque tradition bafouée, mais à mettre en cause la prise en charge des festivités par l'OTCN. Ils dénonçaient l'absence de signification sociale et politique d'une tradition relancée à des fins touristiques et dont l'intérêt pour la municipalité ne résiderait, selon leurs termes, que dans son impact économique et médiatique. Ils affirmaient ne pas se reconnaître dans l'esprit de cette manifestation et dans l'image de Nice, capitale de la Côte d'Azur, dont elle permet d'assurer la promotion touristique: «On ne se retrouvait pas dans cet énorme Club Méditerranée, dans cette orientation qui nous est présentée comme étant inéluctable et qui dévitalise tout lieu de vie pour en faire un décor, qui transforme toute culture en une sorte d'expression figée d'un folklore de pacotille», expliquait l'un de leurs porte-parole sur les ondes d'une radio nationale à l'occasion d'une émission consacrée à la vie culturelle, artistique et politique niçoise.

Cette critique conforte sur certains aspects la distinction entre identité « pour soi » et identité « pour les touristes » en ce qu'elle stigmatise la mise en scène de traditions locales relancées à des fins touristiques. Mais elle dénonce également la posture traditionaliste qui consiste à penser que ce sont les origines locales des « interprètes de la tradition » qui confèrent la légitimité de leur action. Pour les jeunes artistes qui la portent, ce n'est pas parce qu'il est « parisien », étranger à la culture niçoise, que Sergueï suscite l'indignation, mais parce qu'il participe, au même titre que les «carnavaliers » d'antan et les « carnavaleurs » d'aujourd'hui, de ce qui est qualifié de «mascarade touristique». Interrogés sur la 
controverse du « relookage » du Carnaval de Nice, les « francs-tireurs de l'identité locale » comme les appellent les journalistes de la presse régionale, répondent:

Crier au crime de lèse-identité sous le seul prétexte que l'on a modifié l'esthétique des chars ne veut rien dire. Tout au plus, cela démontre que l'on a de l'identité culturelle une vision muséale, figée. L'identité, ça ne se met pas en conserve, ça se vit, ça s’invente au jour le jour. Notre idée, c'était une fête en rupture du carnaval marchandise. Et qu'il soit conçu sur le modèle ancien, façon Mossa, ou revu par Sergueï ne change en rien notre propos ${ }^{20}$.

Ils dénoncent ainsi la mise en spectacle de la ville à des fins touristiques et tentent de mettre en usage des formes festives alternatives et non marchandes.

C’est dans cet esprit que furent créés dès le milieu des années 1990 des carnavals dits « indépendants» dans deux quartiers de Nice situés à la marge des festivités officielles orchestrées par l'отсN. D’abord fréquentés par quelques dizaines de personnes, ils ont été investis par un public de plus en plus nombreux et suscitent désormais le plus grand intérêt des médias qui y voient une alternative incontournable et un complément indispensable aux festivités de la ville. Le principe était d'inventer des carnavals dits « de participation» permettant de réconcilier la population locale avec la notion de fête populaire. Dans cet esprit, les chars et les déguisements devaient être confectionnés à partir de la récupération et du détournement d'objets de consommation accessibles à tout le monde (chariots de supermarché, cartons d'emballage, remorques de bateau, planches à roulettes, palettes de chantier, etc.). Les jets de farine ont également été préférés aux confettis vendus dans les corsos du carnaval « officiel » et se sont rapidement imposés comme une marque distinctive de ces manifestations.

Dès le début, les acteurs des carnavals «indépendants » ont prôné un mode de participation différent de celui des festivités carnavalesques relancées dans les villages de l'arrière-pays niçois grâce aux conseils éclairés des folkloristes ou ethnologues locaux. On n'y rencontre pas de formations musicales qui interprètent un répertoire traditionnel selon les coutumes de l'époque, mais des sound systems ${ }^{21}$ librement inspirés des trios électriques $^{22}$ qui se sont développés ces dernières années dans le carnaval de Bahia. Au-delà de la forme de participation, un style musical s'est également imposé. Il s'agit d'une base rythmique inspirée du raggamuffin jamaïcain et qui a pour caractéristique, contrairement au rock, de donner la priorité à la parole et à l'ambiance festive. Au fil des années, une autre base rythmique a progressivement été introduite dans les corsos. Il s'agit d'une samba d'inspiration afro-brésilienne telle qu'elle est jouée et dansée dans le carnaval de Bahia par les batucadas, ces groupes de percussionnistes et de danseurs de rue qui peuvent parfois atteindre plusieurs centaines de personnes et qui se sont imposés comme le symbole de la résistance culturelle et de l'élaboration d'une identité «afro » dans la société bahianaise (Ribart, 1999; Agier, 2000).

20. Cité dans Nice-Matin du 23 février 2000, «Les “indépendants” comptent les points ».

21. Un sound system est une configuration scénique comprenant le matériel nécessaire à la sonorisation de musiques préenregistrées (platines, lecteur de disques compacts, disques échantillons...) et des micros.

22. Il s'agit de camions à plateformes sonorisées pouvant accueillir les chanteurs de groupes locaux ou invités. 
Au fil des années s'est développé dans ces manifestations de rue tout un imaginaire culturel et pictural dont la symbolique est venue nourrir une vision " anti-Côte d'Azur » de la ville centrée sur la reconquête des espaces de vie en commun. Capitian Nissa est ainsi devenu une sorte de Superman local dont la mission consiste à lutter contre les «estraças terrestras ${ }^{23}$ ", ces hommes et ces femmes venus d'ailleurs, vêtus de shorts et de sandales et qui envahissent les plages en période estivale. Une autre figure emblématique des carnavals indépendants est incarnée par lo Gran Calamar, un céphalopode géant qui recule généralement devant l'avancée des touristes, mais qui, selon la légende, reviendra un jour sur le rivage pour les dévorer. Dans un autre registre, Lo Drag s'est également imposé depuis son apparition en 1998 comme le symbole local de la lutte contre l'exclusion. Il est une sorte de dragon mythique qui, comme l'explique son créateur, est là pour manger tous les $\operatorname{cònòs}^{24}$ de la ville, ceux qui ne tolèrent pas l'altérité et qui ne conçoivent l'identité locale que comme un héritage ne pouvant se transmettre que par filiation. Le cònò est alors celui qui considère que l'on ne peut être niçois que si l'on est «de souche» niçoise, ce qui revient à clôturer le groupe sur la base des origines communes et à exclure tous ceux qui ne remplissent pas ces critères d'appartenance. La formule Siam toi de Nissa ? ${ }^{55}$ érigée en slogan des carnavals indépendants est alors une manière de répondre à la critique traditionaliste principalement portée par l'association Sian de Nissa $!^{26}$, dont le nom même repose sur l'affirmation des origines.

Cette démarche mise en œuvre dans la réalisation des carnavals «indépendants » se traduit également par une critique de la définition traditionaliste de l'authenticité qui prône la conservation de la culture et des «vraies » valeurs d'antan. Se faisant l'héritière des positions associées au mouvement de Mai 68 et à partir desquelles fut élaborée une déconstruction radicale de l'exigence d'authenticité, elle ne pouvait que dénoncer toute approche faisant comme s'il pouvait exister vraiment, quelque part, une authenticité préservée. Se formulant en permanence dans une distance ironique par rapport à elle-même, cette démarche repose alors principalement sur la notion de pantai ${ }^{27}$ et sur l'exaltation du principe d'invention culturelle. Dans ces conditions, face à un carnaval décrit comme un outil commercial et taxé de ce fait d'inauthenticité, c'est l'inventivité elle-même, la capacité des participants de la fête à pantaïer, à créer de nouveaux signes et emblèmes de l'identité locale et non la «tradition », à savoir l'oubli de l'invention, qui permet de garantir l'authenticité de la fête et son caractère identifiant.

23. Estraça ou estras en provençal signifie « chiffon, lambeaux, guenilles...».

24. Ce terme s'est imposé dans toute la région après la sortie du premier album de Massilia Sound System à Marseille et de leur chanson Stop the cònò !

25. «Nous sommes tous de Nice», en français.

26. "Nous sommes de Nice.»

27. Le pantaï est un mot issu du dialecte niçois, proche du provençal, qui n'a pas vraiment d'équivalent en français et qui signifie à la fois le rêve, l'imagination, la créativité. Dans les mouvements alternatifs de la ville, l'expression «avoir le pantaï» renvoie à l'état d'esprit qui permet de donner libre cours à ses phantasmes et qui accorde une large place à la fête et au jeu. Ainsi, les carnavals «indépendants » se définissent comme étant «100\% pantaï» et le journal local à tendance satirique créé par des membres du collectif artistique et culturel a choisi comme slogan la formule «Mas de Pantaï», plus de pantaï. 
C'est précisément dans cet esprit que les carnavals «indépendants » ne constituent plus aujourd'hui qu'un événement parmi d'autres de l'expression de cette critique « artiste» qui s'inscrit désormais dans un ensemble plus vaste d'activités festives. Depuis 1996, ceux-ci sont consignés dans un calendrier de fabrication artisanale vendu dans les lieux alternatifs de la ville. Par exemple, le premier dimanche de février est consacré au festin dei pahlassos qui ouvre la période carnavalesque. Cette tradition inventée - et revendiquée comme telle - s'appuie sur une pratique ancienne que les historiens locaux font remonter à l'époque médiévale et qui consistait à faire sauter un mannequin de paille sur un drap tendu par les pêcheurs du Vieux-Nice. Le pahlasso symbolisait les notables de la ville dont on se vengeait avec humour. Mais, contrairement aux pratiques d'antan, cette tradition inventée se présente sous la forme d'une compétition, le «championnat du monde de lancer de pahlasso » qui consiste, selon l'expression consacrée, à " propulser le plus loin possible le pahlasso qui est en nous». Le prix le plus convoité par les participants - le trophée du meilleur pantaï - est alors, contrairement à toute attente, celui qui sanctionne l'écart le plus remarqué au règlement très pointilleux de l'épreuve que chacun s'applique à rappeler et à faire respecter.

Le $1^{\text {er }}$ mai, c'est un autre personnage mythique, la Santa Capelina, qui est célébré sur le quai de Rauba Capeù dont la vue imprenable sur la Baie des Anges a fait un passage obligé des touristes. Ici, l'association entre la date de l'événement - le $1^{\mathrm{er}}$ mai, jour de la fête du travail - et son lieu - Rauba Capeù (littéralement «où les chapeaux s'envolent ») - a donné naissance à la « fête des travailleurs du chapeau », à savoir, précisément, de ceux qui pantaïent (l'expression «travailler du chapeau» étant une autre traduction possible du verbe niçois pantaïer). D'autres moments marquent ce calendrier festif comme, par exemple, Lo Passagin, une sorte de régate maritime à partir d'embarcations de fortune qui se déroule en période estivale, ou encore le Championnat du monde de Pilo ${ }^{28}$ organisé chaque année dans une commune de l'arrière-pays niçois. À chaque fois, c'est le caractère volontairement inventé et «délirant» de ces manifestations - comme le fait d'organiser des championnats du monde à partir de pratiques locales comme le pilo, voire totalement dérisoires comme le lancer de pahlasso - qui est mis en scène par les participants de manière à bien marquer leurs distances avec les initiatives municipales ou associatives de relance des traditions, souvent soutenues et encouragées par les spécialistes locaux. Ils dénoncent ainsi et tournent en dérision à la fois ceux qui militent pour la sauvegarde du folklore local et ceux qui visent à relancer et à mettre en scène des traditions pour construire une localité touristique. Ils tendent également par là, d'une manière différente de la critique traditionaliste, à produire une autre image normée de la ville, une «anti-Côte d'Azur » à laquelle chacun est supposé pouvoir s’identifier.

28. Le pilo (ou pilou) est un jeu de rue et de cour d'école qui se pratique dans la région depuis la guerre ou l'immédiat après-guerre. Il se joue avec une sorte de volant réalisé à partir d'une ancienne pièce de monnaie trouée et d'un bout de papier, de préférence par équipes de deux contre deux. Relancé en 1987 avec la création de ce championnat du monde, il se veut être un des symboles de l'identité niçoise. Voir A. Giordan et J. Maria (1998). 
Cette analyse du Carnaval de Nice, de ses critiques et de ses manifestations alternatives nous invite à revenir sur la problématique de la fabrique des images identifiantes. L'usage de cette notion nous a permis d'appréhender le spectacle de cette ville à travers sa fête la plus identifiante: Nice étant présentée dans les brochures touristiques comme une «ville de carnaval». Tel qu'il nous est apparu, ce spectacle prend des formes diverses selon que ses différentes mises en scène sont travaillées pour les touristes ou pour la communauté locale, dans des registres propres à la tradition ou à l'innovation culturelle, pour représenter une «identité locale» ouverte sur le monde ou refermée sur elle-même, etc.

C'est en tout cas ce que l'on a pu constater à partir des différentes tensions qui travaillent les fêtes carnavalesques comme «spectacle de l'authentique». On a pu voir par exemple comment celle qui s'exerce entre la valorisation marchande d'une différence mise en scène "pour les touristes » et les exigences de compréhension par des publics non initiés aux singularités locales conduisait à un travail de sélection des signes les plus lisibles, les plus œcuméniques et les mieux à même d'être traduits dans des procédures instituées. On a vu également comment, du côté de la critique, la défense réactive d'un local «pour soi » entraînait ses auteurs dans une autre tension, celle qui distingue les velléités de conservation des attributs figés d'une culture essentialisée des tentatives d'accomplissement d'une identité à partir de connexions sémantiques, de «branchements » sur d'autres cultures mondialisées (Amselle, 2001), et qui revient soit à exclure toute forme d'innovation et d'apports extérieurs soit, au contraire, à devoir sans cesse faire preuve d'inventivité et de mise à distance ironique de ses propres positions sous peine de se voir appliquer à soimême les critiques d'inauthenticité portées aux autres.

Un des effets de ces tensions est qu'elles contribuent au brouillage des signifiants identitaires pris dans les jeux de production, de sauvegarde ou de mise en scène de l'authenticité et à partir desquels s'élaborent les images identifiantes de la ville. En ce sens, les représentations de l'histoire de la ville, de son patrimoine et de son territoire qu'offre ce spectacle de l'authentique brouillent les images plus qu'elles ne permettent à la collectivité locale de se voir dans le miroir de ce qu'elles donnent à voir.

\section{RÉSUMÉ}

En s'appuyant sur la notion d'image identifiante forgée par Marc Augé pour rendre compte des représentations que les collectivités locales donnent à voir de leur histoire, de leur patrimoine et de leur territoire à travers des discours sur «l'identité locale », cet article s'intéresse au Carnaval de Nice tel qu'il est impliqué depuis plus d'un siècle dans la construction touristique de l'image de la ville, à ses reformulations successives, à ses critiques ainsi qu'aux manifestations alternatives qui se sont développées ces dernières années sous la forme de «carnavals indépendants ». L'analyse souligne alors les tensions autour desquelles s'articulent les différents points de vue qui travaillent ces festivités comme «spectacle de l'authentique».

\section{ABSTRACT}

Based on the notion of the identifying image developed by Marc Augé to account for the representations that local communities offer of their history, heritage and territory through discourses on "local identity", this paper studies the Carnival of Nice and its involvement for 
over a century in the tourist-based construction of that city's image in its successive reformulations and critiques as well as in the alternative events that have developed over the past few years in the form of "independent carnivals." The analysis then highlights the tensions surrounding the various points of view that strive to present these festivities as a "spectacle of the authentic."

\section{RESUMEN}

Apoyándose en el concepto de imagen identificadora elaborado por Marc Augé para dar cuenta de las representaciones que muestran las colectividades locales de su historia, su patrimonio y su territorio a través de los discursos sobre "la identidad local", este artículo se interesa por el Carnaval de Niza tal como se presenta desde hace más de un siglo en la construcción turística de la imagen de la ciudad, de sus reformulaciones sucesivas, de sus críticas así como de las manifestaciones alternativas que se han desarrollado estos últimos años bajo la forma de "carnavales independientes". El análisis destaca entonces las tensiones alrededor de las cuales se articulan los diferentes puntos de vista que trabajan estas festividades como "espectáculo de lo auténtico".

\section{BIBLIOGRAPHIE}

Agier, M. (2000), Anthropologie du carnaval. La ville, la fête et l'Afrique à Bahia, Paris, Éditions Parenthèses. Augé, M. (1994), Pour une ethnographie des mondes contemporains, Paris, Aubier.

Amselle, J.-L. (2001), Branchements. Anthropologie de l'universalité des cultures, Paris, Flammarion.

Baкнтіne, M. (1970), L'ouvre de François Rabelais et la culture populaire au Moyen Âge et sous la Renaissance, Paris, Gallimard.

Boltanski, L. et E. Chiapello (1999), Le nouvel esprit du capitalisme, Paris, Gallimard.

Bourdin, A. (2000), La question locale, Paris, PUF.

Cousin, S. (2003), L'identité au miroir du tourisme. Usages et enjeux des politiques de tourisme culturel, Thèse de doctorat en anthropologie sociale et ethnologie, EHESS-Paris, Paris.

Davault, C. (2000), «De Nantes la bleue à Saint-Denis la rouge : désindustrialisation, délocalisations et invention de traditions locales", Les traditions inventées. Bilan et perspectives d'un paradigme, Nice, Colloque du SOLIIS-URMIS.

Fighiera, C.-A. (1973), «Histoire du Carnaval de Nice», in Batier (Éd.), Sa Majesté Carnaval, Nice, 18731973, Nice, Imprimerie Meyerber.

Fighiera, C.-A. (1985), «Les origines du Carnaval de Nice», Le Carnaval, la fête et la communication, Nice, Éditions Serre, p. 139-141.

GARAT, I. (1994), «Vivre Bayonne intensément. Mise en scène de l'identité et de la citoyenneté urbaine à travers la fête ", Les cahiers du LERASS, $\mathrm{n}^{\circ}$ 31, p. 109-124.

Giordan, A. et J. Maria (1998), Et vive le pilou! E viva lo polo!, Nice, Z’Éditions.

Hannerz, U. (1996), Transnational Connections: Culture, People, Places, London, Routledge.

Lextrait, F. (2001), Une nouvelle époque de l'Action Culturelle, Rapport à Michel Duffour, Secrétaire d'État au Patrimoine et à la Décentralisation culturelle.

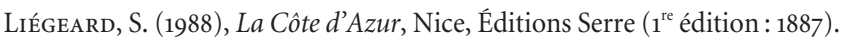

Ribart, F. (1999), Le carnaval noir de Bahia. Ethnicité, identité, fête afro à Salvador, Paris, L'Harmattan.

Sidro, A. (1979a), Le Carnaval de Nice et ses Fous, Nice, Éditions Serre.

Sidro, A. (1979b), Triboulet au royaume de Carnaval. Histoire psychosociale du Carnaval de NICE (1873-1979), Thèse de troisième cycle, Université de Nice. 